\title{
Creating and maintaining a diverse pipeline: a center-wide model and its application to individual laboratories.
}

\author{
William J. Bauer ${ }^{a, b}$, BioXFEL Education and Diversity Committee ${ }^{b}$ \\ ${ }^{a}$ Hauptman-Woodward Medical Research Institute, 700 Ellicott Street, Buffalo, New York \\ 14203, USA, wbauer@hwi.buffalo.edu, ${ }^{b}$ BioXFEL Science and Technology Center, NSF Award \\ 1231306
}

The importance of a creating a diverse environment has been well established and recognized as beneficial to the workplace ${ }^{1}$. More diverse workforces have the potential to be more competitive, innovative, and profitable. To leverage this advantage, many funding agencies require proposals to include a diversity integration component, however they typically provide limited guidance on the design and implementation strategies. As Associate Education and Diversity Director for an NSF Science and Technology Center (STC), BioXFEL, I have designed a multi-institution pipeline that leverages existing infrastructure and has created new mechanisms to incorporate underrepresented populations. This model is customized to meet the needs of our participants, but also addresses issues common among underserved groups. I will describe some of the best practices used by our Center, as well as other STCs, to recruit, prepare, retain, and advance the scientific careers of underserved individuals. The model presented here will include specific examples of successful diversity initiatives that integrate with the scientific and education programs of the Center as well as recommendations on how these can be applied to the individual lab setting.

1. Hunt V., Prince S., Dixon-Fyle S., Lareina Y., Delivering through Diversity, January 2018. McKinsey \& Company.

2. Hunt V., Layton D., and Prince S. Why Diversity Matters, January 2015. McKinsey \& Company. 